\title{
Routine HIV testing in the context of syndromic management of sexually transmitted infections: outcomes of the first phase of a training programme in Botswana
}

\author{
M R Weaver ${ }^{1,2}$ M Myaya, ${ }^{2}$ K Disasi, ${ }^{3}$ M Regoeng, ${ }^{4}$ H N Matumo, ${ }^{4}$ M Madisa, ${ }^{2}$ \\ N Puttkammer, ${ }^{1,2}$ F Speilberg, ${ }^{6}$ P H Kilmarx, ${ }^{3,5}$ J M Marrazzo ${ }^{1}$
}

${ }^{1}$ University of Washington,

Seattle, Washington, USA;

${ }^{2}$ International Training and

Education Center on HIV

(I-TECH), Seattle, Washington,

USA; ${ }^{3}$ BOTUSA (United States

Centers for Disease Control and

Prevention (CDC)/Botswana),

Gaborone, Botswana; ${ }^{4}$ Ministry

of Health of the Republic of

Botswana, Gaborone, Botswana;

${ }^{5}$ National Center for HIV/AIDS,

Viral Hepatitis, STD, and TB

Prevention, CDC, Atlanta,

Georgia, USA; ${ }^{6}$ University of

California at San Francisco, San

Francisco, California, USA

Correspondence to:

Dr M R Weaver, Department of Health Services and I-TECH,

University of Washington, 901

Boren, Suite 1100, Seattle, WA

98104,USA; mweaver@

u.washington.edu

Accepted 16 January 2008

\section{UNLOCKED}

This paper is freely available online under the BMJ Journals unlocked scheme, see http:// sti.bmj.com/info/unlocked.dtl

\section{ABSTRACT}

Objective: In 2004, the Ministry of Health adopted revised protocols for the syndromic management of sexually transmitted infections (STI) that included routine HIV testing. A training programme for providers was developed on the revised protocols that featured interactive case studies and training videos. An objective of the first phase of the training programme was to test its effect on four measures of clinical practice: (1) routine HIV testing; (2) performance of physical examination; (3) risk-reduction counselling and (4) patient education.

Methods: Clinical practice in a district where providers were trained was compared with a district without training. The measures of clinical practice were reported by 185 patients of providers who had been trained and compared with reports by 124 patients at comparison clinics.

Results: Relative to patients at comparison clinics, a higher percentage of patients of trainees reported that the provider: (1) offered an HIV test (87\% versus $29 \%$; $p<0.001)$; (2) conducted a physical examination $(98 \%$ versus $64 \% ; \mathrm{p}<0.001)$; (3) helped them to make a plan to avoid future STI acquisition $(95 \%$ versus $76 \%$; $p<0.001)$ and (4) provided patient-specific information about HIV risk (65\% versus $32 \% ; p<0.001)$. Among patients offered HIV testing, the percentage who accepted did not differ between groups (38\% of 161 patients of trainees versus $50 \%$ of 36 comparison patients; $p=0.260$ ). Overall, $33 \%$ of patients of trainees and $14 \%$ of comparison patients were tested $(p<0.001)$. Conclusion: A multifaceted training programme was associated with higher rates of HIV testing, physical examination, risk-reduction counselling and better HIV risk education.

Several African countries have adopted syndromic management for sexually transmitted infections (STI) as part of their comprehensive reproductive health, STI and HIV prevention strategies. ${ }^{1-7}$ Syndromic management is based on a presumptive diagnosis of STI and is typically directed by national protocols for treatment based on symptoms and easily recognised signs of infection. ${ }^{8}$ Regardless of whether the diagnosis is presumptive or aetiological, correct case management of STI includes nine elements: history; physical examination; diagnosis; early and effective treatment; advice on sexual behaviour; promotion and provision of condoms; partner notification and treatment; case reporting and, if necessary, clinical follow-up. ${ }^{8}$
In Botswana, the Ministry of Health (MOH) reviews and updates its national syndromic management protocols based on periodic aetiological studies and other developments in healthcare. ${ }^{9-11} \mathrm{~A}$ study performed in 2002 that used highly sensitive diagnostic assays demonstrated a high prevalence of HIV among patients seeking care for STI-related complaints relative to a sentinel HIV prevalence of $38.6 \%$ among pregnant women in Botswana in $2001 .^{12}$ HIV prevalence was $54 \%$ among women with vaginal discharge or lower abdominal pain (VD/LAP), 62\% among men with urethral discharge and $74 \%$ among patients with genital ulcer disease (GUD). ${ }^{11}$ The proportion of GUD cases caused by genital herpes increased between 1993 and 2001, whereas the proportion caused by syphilis and chancroid decreased. ${ }^{11}$

In 2004, the $\mathrm{MOH}$ adopted revised protocols that included routine HIV testing as part of every STI consultation. Botswana began routine, noncompulsory HIV testing (ie, "opt-out") as part of all medical services in January $2004^{13} 14$ and revised protocols presented an opportunity to integrate STI and HIV care further. In the revised GUD protocol, patients with GUD receive episodic treatment for genital herpes with acyclovir (400 mg by mouth three times a day for seven days) as well as treatment for syphilis and chancroid; patients whose GUD is characterised by vesicles receive only episodic treatment with acyclovir.

To implement the revised protocols, the $\mathrm{MOH}$ developed a new national STI training programme in cooperation with the BOTUSA (United States Centers for Disease Control and Prevention (CDC)/Botswana) and the International Training and Education Center on HIV/AIDS (I-TECH). An objective of the first phase of the training programme was to test its effect on four measures of clinical practice: (1) routine HIV testing; (2) performance of physical examination; (3) riskreduction counselling and (4) patient education.

\section{METHODS}

\section{Study design}

Clinical practice in a district where providers were trained was compared with a district without training. Several weeks after training, its effects on clinical practice were measured by patient reports during exit interviews after visits that included STI care. A trained interviewer read standardised 
questions to the patient about whether or not tasks were performed by providers. The interviewer also read statements about the quality of care and asked the patient to rate the care on a five-point scale, in which "strongly agree" was rated 5 and "strongly disagree" was rated 1.

\section{Selection of districts}

The selection of the training (Lobatse Town Council) and comparison (Southeast) districts was based on four criteria: (1) average or higher than average proportion of GUD cases among STI visits; (2) low number of clinics; (3) clinics located relatively close to one another within a district and (4) proximity of districts. The number of clinics was a criterion because providers were trained by district; only approximately 200 people could be trained in the first phase and the low number of clinics corresponded to the low number of people to train per district. Twenty-three per cent of STI visits were for GUD in Lobatse and $17 \%$ in Southeast compared with a national average of $17 \%$. Lobatse had nine public clinics and Southeast had 11 compared with a national average of 26 . Clinics were located relatively close to one another in both districts. Lobatse and Southeast had a higher-than-average population density (703 and 34 people per square kilometre compared with a national average of three). ${ }^{15}$ Lobatse and Southeast are geographically adjacent, which may have reduced differences among clinics and in health-seeking behaviour between the two patient populations.

\section{Selection of clinics}

Clinics were selected on the basis of two criteria: (1) an average of 10 or more STI cases per month and (2) clinics at which patients could give informed consent. Patients were interviewed at five of the nine facilities in Lobatse, including outpatient clinics of the district hospital and four public sector primary care clinics. Four facilities were excluded: a mental health hospital, a prison clinic and two clinics that reported fewer than 10 STI cases per month. In Southeast district, patients were interviewed at the hospital of a faith-based organisation and six of 11 public health facilities, including a primary care clinic and five health posts, which are the smallest unit of Botswana's primary care system. Five facilities that reported fewer than 10 STI cases per month were excluded.

\section{Intervention}

All nurses, nurse midwives and medical officers in Lobatse Town Council were entitled to training and 194 out of 212 providers (91.5\%) were trained during 11 three-day sessions from 6 September to 28 October 2004. The curriculum was designed for clinical training in resource-limited settings, including interactive case studies and films on patient-centered care, sensitive female pelvic examination, risk-reduction counselling and HIV post-test counselling (available at http://www. go2itech.org). ${ }^{16-19}$ Sessions were facilitated by master trainers from the National STI Training and Research Center and core trainers, who were trained as trainers in the new curriculum.

\section{Recruitment of patients}

Female patients 16-49 years old presenting with GUD and/or VD/LAP and male patients 16-49 years old presenting with GUD and/or urethral discharge were eligible. Providers referred all patients who met the inclusion criteria to an interviewer. Participants provided informed consent by signing, initialing or marking a consent form that was countersigned by the interviewer. An exception was when a clinical specialist was at clinics in Lobatse to observe visits (see "Statistical analysis") and countersigned the consent form.

A total of 216 patients in Lobatse and 128 patients in Southeast district were invited to participate; response rates were $86 \%$ and $97 \%$, respectively (185 patients in Lobatse and 124 patients in Southeast district).

\section{Human subjects review}

All procedures and questionnaires were reviewed and approved by the Botswana $\mathrm{MOH}$ Health Research Unit. They were also reviewed by the Associate Director for Science, National Center for HIV, STD and TB Prevention, CDC, who determined that the activity was not research.

\section{Statistical analysis}

Bivariate analyses were conducted using SPSS-PC version 13.0 for Windows (SPSS Inc, Chicago, Illinois, USA). All $p$ values were two-sided and a level of less than 0.05 was considered significant. The relative risk was calculated as the ratio of training to comparison clinic percentages.

When there were differences between training and comparison samples, multivariate analyses were performed with a modified Poisson regression approach ${ }^{20}{ }^{21}$ using SAS software version 9.1 (SAS Institute Inc, Cary, North Carolina, USA) and the adjusted relative risk is reported. The visits of approximately half the patients at training clinics were observed by a clinical specialist. An observer could have improved the providers' practice during those visits, thus the multivariate analysis included the presence of the observer as a variable.

The dependent variables for the 11 statements about quality of care were analyzed two ways: (1) as a dichotomous choice between agreement or disagreement with the statement or (2) as a continuous response on a five-point scale. The results of the analyses were similar and the former is reported.

To facilitate interpretation, STI syndrome variables were hierarchical; for example a patient who reported both GUD and VD/LAP was only counted as GUD. Only 23 out of 194 women (14\%) with VD/LAP also reported GUD. Only eight out of 36 men $(18 \%)$ with urethral discharge also reported GUD.

\section{RESULTS}

\section{Patient and provider characteristics}

The characteristics of patients who were interviewed and their visits are presented in table 1 . Significantly more patients at training clinics were treated by nurse midwives (31\%) than at comparison clinics $(16 \%)(p=0.003)$ and correspondingly fewer by nurses. Significantly more patients had visits for follow-up care at training clinics than comparison clinics (29\% versus $10 \%$, respectively, $\mathrm{p}<0.001$ ) and correspondingly fewer were first visits. The syndromes that patients reported were similar, with one exception. At training clinics, $8 \%$ of the patients reported urethral discharge compared with $18 \%$ at comparison clinics ( $p=0.006$ ) or $27 \%$ compared with $51 \%$ of the male patients, respectively.

\section{Routine HIV testing}

Significantly more patients at training clinics had an HIV test $(33 \%)$ than at comparison clinics $(14 \%)(p<0.001)$. As shown in fig $1,91 \%$ of the patients at training clinics reported having enough opportunity to talk privately with the provider about HIV testing compared with $33 \%$ at comparison clinics $(p<0.001)$ and $87 \%$ of the patients at training clinics were offered an HIV test compared with $29 \%$ at comparison clinics 
Table 1 Description of patients and their visits at clinics with the STI syndromic management training programme and comparison clinics, Botswana 2004

\begin{tabular}{|c|c|c|c|}
\hline & $\begin{array}{l}\text { Patients from } \\
\text { clinics with trained } \\
\text { health workers }\end{array}$ & $\begin{array}{l}\text { Patients from } \\
\text { comparison clinics }\end{array}$ & \\
\hline & n (\%) & n (\%) & p Value \\
\hline \multicolumn{4}{|l|}{ Characteristics of patients } \\
\hline Gender of patient (\% female) & $134(74)$ & $81(65)$ & 0.119 \\
\hline Average age in years & 28 & 29 & 0.286 \\
\hline \multicolumn{4}{|l|}{ Language spoken at home } \\
\hline Setswana & $173(94)$ & $111(90)$ & 0.207 \\
\hline Kalanga & $2(1)$ & $6(5)$ & 0.064 \\
\hline English & $5(3)$ & $2(2)$ & 0.706 \\
\hline Other & $5(3)$ & $5(4)$ & 0.530 \\
\hline \multicolumn{4}{|l|}{ Education (one missing from each district) } \\
\hline No formal, non-formal and primary & $50(27)$ & $38(31)$ & 0.490 \\
\hline Junior secondary & $78(42)$ & $54(44)$ & 0.809 \\
\hline Senior secondary and post-secondary & $56(30)$ & $31(25)$ & 0.313 \\
\hline Married or cohabiting & $54(29)$ & $41(33)$ & 0.469 \\
\hline \multicolumn{4}{|l|}{ Characteristics of patients' visits } \\
\hline Gender of provider who treated patient ( $\%$ female) & $153(83)$ & $94(76)$ & 0.138 \\
\hline \multicolumn{4}{|l|}{ Profession of provider who treated patient } \\
\hline Nurse & $122(66)$ & $101(82)$ & 0.003 \\
\hline Nurse midwife & $57(31)$ & $20(16)$ & 0.003 \\
\hline Medical officer & $6(3)$ & $3(2)$ & 0.745 \\
\hline \multicolumn{4}{|l|}{ Visit type } \\
\hline First visit & $132(71)$ & $111(90)$ & 0.001 \\
\hline Follow-up visit & $53(29)$ & $13(10)$ & $<0.001$ \\
\hline \multicolumn{4}{|l|}{ Syndromes } \\
\hline Genital ulcer & $45(24)$ & $28(23)$ & 0.724 \\
\hline VD/LAP & $109(59)$ & $62(50)$ & 0.122 \\
\hline Urethral discharge & $14(8)$ & $22(18)$ & 0.006 \\
\hline No symptoms & $17(9)$ & $12(10)$ & 0.885 \\
\hline Visit observed by clinical specialist & $98(53)$ & $0(0)$ & $<0.001$ \\
\hline Referred by contact slip & $14(8)$ & $6(5)$ & 0.480 \\
\hline Sample size & 185 & 124 & \\
\hline
\end{tabular}

STI, Sexually transmitted infection; VD/LAP, vaginal discharge or lower abdominal pain.

$(\mathrm{p}<0.001)$. Among patients offered an HIV test, 38\% of 161 at training and $50 \%$ of 36 at comparison clinics $(p=0.260)$ agreed to be tested.

A multivariate analysis adjusted for the differences between samples in provider profession, visit type, patient gender, syndromes, observation by a clinical specialist and before coming to the clinic whether or not the patient understood that $\mathrm{s} /$ he had the right to refuse an HIV test. As shown in columns 2 and 3 of table 2, patients at training clinics were 2.55 (95\% confidence interval (CI) 1.96 to 3.32 ) times more likely than at comparison clinics to talk privately with a provider about HIV testing. As shown in columns 4 and 5 , patients at training clinics were 2.92 (95\% CI 2.19 to 3.90) times more likely than at comparison clinics to be offered an HIV test.

The percentage of patients who refused an HIV test because they had already had one was similar in both samples; $29 \%$ for training and $28 \%$ for comparison clinics $(p=0.581)$. When these patients were omitted from the sample, the results reported above did not change substantially.

\section{Physical examination}

Patients at training clinics were significantly more likely to report having a physical examination than at comparison clinics (98\% versus $64 \%$, respectively, $\mathrm{p}<0.001$ ). Multivariate comparisons also showed significant differences between samples; patients at training clinics were 1.52 (95\% CI 1.34 to 1.73 ) times more likely than at comparison clinics to have a physical examination.

\section{Patient satisfaction with quality of care}

Patients at training clinics rated the quality of care more highly than at comparison clinics. After adjusting for differences between training and comparison clinics (columns 6 and 7 of table 3), the relative risk was significantly higher that patients at training than comparison clinics would strongly agree or agree with the following statements: (1) Did you feel the health worker gave you treatment for your problem?; (2) Did the health worker give you information about the nature of your problem?; (3) Did the health worker help you make a plan so that you could better prevent this problem in the future? and (4) On the whole, were you satisfied with the care you received for your problem today?

\section{Patient education}

Patients at training clinics were significantly more likely to report what they had learned about their HIV risk from the provider than at comparison clinics $(65 \%$ versus $32 \%$, respectively, $p<0.001)$. Patients were classified as reporting no information from the provider when they said that they did not know or did not remember what the provider said, or that 
Table 2 Modified Poisson regression of factors associated with HIV test outcomes among patients treated for STI, Botswana 2004

\begin{tabular}{|c|c|c|c|}
\hline \multirow[b]{2}{*}{ Independent variables (1) } & \multirow{2}{*}{$\begin{array}{l}\text { Talked privately } \\
\text { with provider } \\
\text { about HIV test* } \\
\begin{array}{l}\text { Relative risk (2) } \\
\left(95 \% \mathrm{CI}^{*}(3)\right)\end{array}\end{array}$} & \multirow{2}{*}{$\begin{array}{l}\text { Offered HIV test* } \\
\text { Relative risk (4) } \\
\left(95 \% \mathrm{CI}^{*}(5)\right)\end{array}$} & \multirow{2}{*}{$\begin{array}{l}\text { Accepted HIV test* } \\
\text { Relative risk (6) } \\
\left(95 \% \mathrm{CI}^{*}(7)\right)\end{array}$} \\
\hline & & & \\
\hline \multicolumn{4}{|l|}{ Training clinic } \\
\hline No $(n=124)$ & Reference & Reference & Reference \\
\hline Yes $(n=185)$ & 2.55 (1.96 to 3.32 ) & $2.92(2.19$ to 3.90$)$ & 0.88 (0.58 to 1.35$)$ \\
\hline Nurse midwife $(\mathrm{n}=77)$ & $1.17(1.04$ to 1.32$)$ & $1.16(1.01$ to 1.33$)$ & 0.99 (0.68 to 1.44$)$ \\
\hline Medical officer $(\mathrm{n}=9$ ) & $1.00(0.68$ to 1.46$)$ & $0.86(0.48$ to 1.54$)$ & $1.16(0.45$ to 3.03$)$ \\
\hline \multicolumn{4}{|l|}{ Visit type } \\
\hline First visit $(\mathrm{n}=229)$ & Reference & Reference & Reference \\
\hline Follow-up visit $(\mathrm{n}=66$ ) & $0.97(0.84$ to 1.11$)$ & $1.03(0.88$ to 1.20$)$ & $0.60(0.36$ to 1.01$)$ \\
\hline \multicolumn{4}{|l|}{ Patient gender } \\
\hline $\operatorname{VD} / L A P(n=171)$ & $0.91(0.76$ to 1.08$)$ & $0.91(0.73$ to 1.14$)$ & $0.83(0.50$ to 1.40$)$ \\
\hline Urethral discharge $(\mathrm{n}=36)$ & $1.06(0.80$ to 1.41$)$ & $1.01(0.75$ to 1.35$)$ & $0.97(0.57$ to 1.64$)$ \\
\hline No symptoms $(\mathrm{n}=29$ ) & $0.89(0.71$ to 1.13$)$ & $0.89(0.68$ to 1.16$)$ & $0.72(0.34$ to 1.49$)$ \\
\hline \multicolumn{4}{|l|}{ Visit observed by clinical specialist } \\
\hline No $(n=211)$ & Reference & Reference & Reference \\
\hline Yes (n = 98) & 1.08 (0.98 to 1.20$)$ & $0.97(0.87$ to 1.10$)$ & $0.89(0.60$ to 1.32$)$ \\
\hline \multicolumn{4}{|l|}{ Understood right to refuse test } \\
\hline No $(\mathrm{n}=99)$ & Reference & Reference & Reference \\
\hline Yes $(n=210)$ & 1.19 (1.02 to 1.39$)$ & $1.14(0.96$ to 1.36$)$ & 0.91 (0.62 to 1.34$)$ \\
\hline Sample size & 305 & 304 & 193 \\
\hline
\end{tabular}

STI, Sexually transmitted infection; VD/LAP, vaginal discharge or lower abdominal pain.

* Modified Poisson regressions adjust for health worker profession (nurse, nurse midwife or medical officer), follow-up visit, patient gender, syndromes, observation by a clinical specialist and before coming to the clinic whether or not the patient understood that s/he had the right to refuse an HIV test. † Results with a $p$ value less than 0.05 are highlighted in bold.

the provider did not discuss the topic. The multivariate comparisons showed that patients at training clinics were 1.72 (95\% CI 1.22 to 2.40 ) times more likely than at comparison clinics to report receiving information about the risk of HIV.

\section{DISCUSSION}

Botswana's new training programme for the revised syndromic management protocols was associated with significant improvements in four outcomes: (1) routine HIV testing; (2) physical

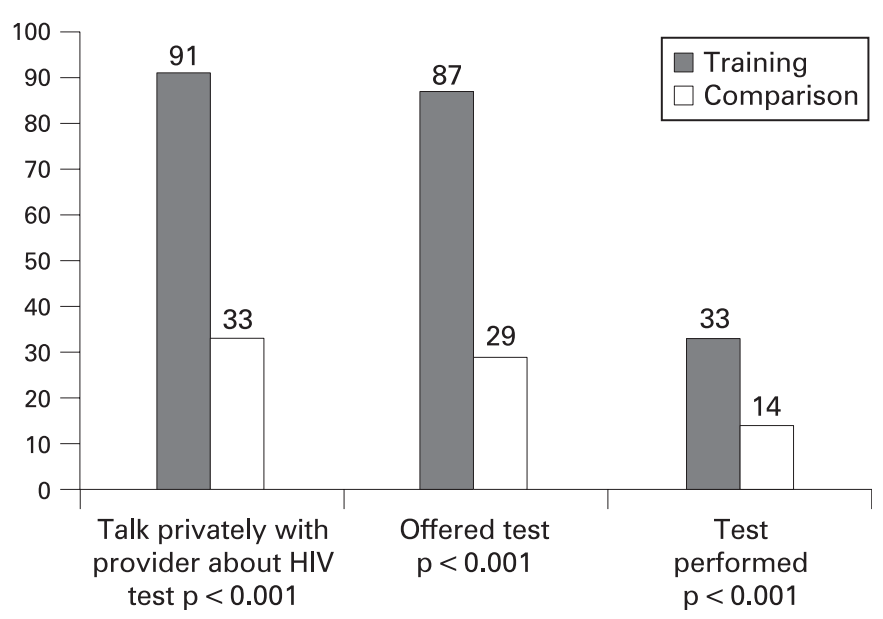

Figure 1 Patient reports on HIV testing at clinics with a sexually transmitted infection syndromic management training programme and comparision clinics, Botswana 2004. examination; (3) risk-reduction counselling and (4) patient education about HIV risk. The providers were significantly more likely to offer an HIV test and overall patients were significantly more likely to have an HIV test at training than at comparison clinics. The likelihood of accepting an HIV test among patients who were offered one was the same for both samples, so having an HIV test depended on whether or not a provider offered it.

This is among the first reports of routine HIV testing for STI patients in a resource-limited setting since June 2004, when the World Health Organisation and the Joint United Nations Programme on HIV/AIDS recommended routine HIV testing in STI clinics or other clinics that provide STI care. ${ }^{22}$ In Botswana, the difference in the percentage of patients who had an HIV test between training (33\%) and comparison clinics (14\%) was within the range reported in England by Day et al. ${ }^{23}$

Providers who attended the new STI training programme were significantly more likely to conduct a physical examination, help patients to make a plan to avoid acquiring STI in the future and discuss HIV risks with patients. Our findings add substantially to the relatively limited body of research demonstrating an effect of STI training in resource-limited settings on conducting a physical examination ${ }^{17}$ and counselling STI patients on their HIV risk. ${ }^{5} 24$

This study is among the first to use patient reports on whether or not tasks were performed by providers. Although patient exit interviews are a well-known method for evaluating the quality of STI care, ${ }^{25}$ previous researchers have only used them to collect information on patient education, ${ }^{76} 27$ opinions on waiting time and satisfaction with care, ${ }^{27}$ condoms $^{76}$ and 
Table 3 Percentage of patients who strongly agreed or agreed with quality of care statements at clinics with the STI syndromic management training programme and comparison clinics, Botswana 2004

\begin{tabular}{|c|c|c|c|c|c|}
\hline \multirow[b]{2}{*}{ Statement (1) } & \multicolumn{4}{|c|}{ Bivariate results } & \multirow{2}{*}{$\begin{array}{l}\text { Multivariate results } \\
\text { Adjusted relative risk } \\
\text { (6) }(95 \% \mathrm{Cl}(7))\end{array}$} \\
\hline & $\begin{array}{l}\text { Training (\%) } \\
(2)\end{array}$ & $\begin{array}{l}\text { Comparison (\%) } \\
\text { (3) }\end{array}$ & $\begin{array}{l}\text { p Value } \\
\text { (4) }\end{array}$ & $\begin{array}{l}\text { Relative risk } \\
(2) /(3)\end{array}$ & \\
\hline $\begin{array}{l}\text { Did you believe that the information you shared } \\
\text { about yourself with the health worker would be kept } \\
\text { confidential? }\end{array}$ & 92 & 87 & 0.120 & 1.06 & $1.04(0.95$ to 1.15$)$ \\
\hline $\begin{array}{l}\text { Did the health worker give you enough opportunity to } \\
\text { explain your problem? }\end{array}$ & 98 & 99 & $0.652 \dagger$ & 0.99 & $1.00(0.98$ to 1.02$)$ \\
\hline $\begin{array}{l}\text { Did you feel comfortable asking the health worker } \\
\text { questions about your problem? }\end{array}$ & 95 & 88 & 0.020 & 1.08 & $1.07(0.98$ to 1.17$)$ \\
\hline $\begin{array}{l}\text { Did you feel comfortable talking about your sexual } \\
\text { behaviours with the health worker? }\end{array}$ & 96 & 89 & 0.035 & 1.08 & $1.04(0.95$ to 1.13$)$ \\
\hline $\begin{array}{l}\text { Did you feel comfortable sharing information about } \\
\text { your sexual partner(s) with the health worker? }\end{array}$ & 93 & 84 & 0.006 & 1.11 & $1.08(0.96$ to 1.20$)$ \\
\hline $\begin{array}{l}\text { Do you believe the health worker accurately identified } \\
\text { your problem? }\end{array}$ & 92 & 86 & 0.049 & 1.07 & $1.06(0.96$ to 1.17$)$ \\
\hline $\begin{array}{l}\text { Did you believe the health worker gave you treatment } \\
\text { for your problem? }\end{array}$ & 83 & 67 & 0.001 & 1.24 & $1.20(1.02$ to 1.40$)$ \\
\hline $\begin{array}{l}\text { Did the health worker give you information about } \\
\text { the nature of your problem? }\end{array}$ & 90 & 72 & $<0.001$ & 1.25 & $1.16(1.01$ to 1.34$)$ \\
\hline $\begin{array}{l}\text { Did the health worker help you make a plan so that } \\
\text { you could better prevent this problem in the future? }\end{array}$ & 95 & 76 & $<0.001$ & 1.25 & $1.21(1.08$ to 1.35$)$ \\
\hline $\begin{array}{l}\text { On the whole, were you satisfied with the care you } \\
\text { received for your problem today? }\end{array}$ & 94 & 87 & 0.034 & 1.08 & $1.10(1.02$ to 1.19$)$ \\
\hline Sample size & 185 & 124 & & & \\
\hline
\end{tabular}

\footnotetext{
STI, Sexually transmitted infection.

* Modified Poisson regressions adjust for health worker profession (nurse versus nurse midwife), follow-up visit, syndromes and observation by a clinical specialist.

$\dagger$ Test statistic is from a Fisher's exact test, because some of the cells in this analysis have an expected frequency of less than five.
}

contact slips. ${ }^{26}$ Some researchers consider unannounced (or blinded) standardised patient encounters to be the "gold standard" for measuring the quality of clinical practice. ${ }^{28}$ Standardised patients can not be used, however, to evaluate some aspects of quality, such as physical examination and the diagnosis of an STI based on clinical symptoms.

The study had several limitations. First, the design was limited to a single time period after training providers at the training sites, so differences between districts could potentially have been confounded with the effects of the training programme. Health districts were, however, selected to minimise differences among clinics and patients and multivariate analyses adjusted for differences between the samples. Second, it is possible that patient visits at training clinics that were not observed were influenced by the recent presence of a clinical specialist. The clinical specialist, however, was not at training clinics on the days when observations were not performed. Third, the analysis may not have fully adjusted for unobserved differences among providers and clinics; random effects analysis of variance regressions would be necessary to adjust fully for these differences. Given the magnitude of the differences between patient reports at training and comparison clinics, however, it is unlikely that the additional analysis would alter the conclusions for the four main outcomes. Fourth, HIV testing results do not include whether or not patients learned their

\section{Key messages}

- The STI training programme for providers was associated with higher rates of HIV testing, physical examination, risk reduction counselling and better HIV risk education.

- Having an HIV test depended on whethor or not the provider offered it.

- Patient reports can be used to evaluate whether or not tasks were performed by providers.
HIV test results. Fifth, the data were collected within two months of training and can not show whether or not the effects of training persisted over time. Future activities will include mentoring and supervision visits for trainees to reinforce the training programme and learn whether or not the results persisted. Finally, the percentage of patients who strongly agreed or agreed with statements about quality of care was high, which could accurately reflect patient experience or could reflect acquiescent response bias. ${ }^{29}{ }^{30}$ To the extent that bias existed, it would not be more likely to occur in training than comparison clinics.

Future studies on the outcomes of training programmes should have a more rigorous quasi-experimental design, with baseline and post-training data. In these studies, the provider or clinic should be the unit of analysis, with a sample of a relatively large number of providers or clinics and a small number of patients per provider or clinic. The analysis should account for clustering at the provider and clinic level.

In conclusion, a multifaceted training programme was associated with higher rates of HIV testing, physical examination, risk-reduction counselling and better HIV risk education.

Acknowledgements: The authors gratefully acknowledge the work of a Botswanabased reference group that guided this study. Reference group members included: Drs Marina Anderson, Ntomdizodwa Bhala and Fatma Hussein and B L Shaw of the AIDS/ STD Unit, MOH; Henry U Iwebor, Central Medical Stores; Dr Joyce Kgatlwane, Botswana Essential Drugs Action Programme; Dr M B Mohammed, Athlone Hospital; Dr B Sikwa, BOMAID; Duncan Thela, Associated Fund Administrator; Dr Tsadik, Lobatse Town Council. The authors also wish to thank the following people for their essential contributions to designing the training programme and support in carrying out the study: Kgoroletso Molosiwa, Chief Health Officer, AIDS/STD Unit, MOH; Mafizur Rahman, former coordinator of the National STI Training and Research Center; Gabriela Paz-Bailey and Caroline Ryan, CDC; Majid Sadigh, Yale University; Erin Seiler and Sara Teitelman, I-TECH; Carolyn Wilson, Vanessa Beyleveld and Dr Muntanga Mapani, Premiere Personnel. They would especially like to thank Doug Fleming of BOTUSA, Naomi Bock, Janet Moore and an anonymous statistical reviewer at the CDC for comments on the manuscript, Bobbi Nodell of I-TECH for editing the manuscript, Michael Chapko of the University of Washington for statistical advice and two anonymous reviewers for valuable comments. 
Funding: The Ministry of Health of Botswana and I-TECH undertook this work with financial and technical assistance from the BOTUSA, which is a partnership between the government of Botswana and the United States Centers for Disease Control and Prevention. I-TECH is funded by the United States Health Resource Services Administration, grant number 5 U69HA00047-04-00.

\section{Competing interests: None.}

Disclaimer: The opinions expressed by the authors contributing to this article do not necessarily reflect the opinions of the United States Department of Health and Human Services, the Public Health Service, the Centers for Disease Control and Prevention or the authors' affiliated institutions. The use of trade names is for identification only and does not imply endorsement by any of the groups named above.

\section{REFERENCES}

1. Boonstra E, Lindbaek M, Koulman E, et al. Syndromic management of sexually transmitted diseases in Botswana's primary healthcare: quality of care aspects. Trop Med Int Health 2003;8:604-14.

2. Voeten HACM, Otido JM, O'Hara HB, et al. Quality of sexually transmitted disease management in Nairobi, Kenya: a comparison among different types of health care facilities. Sex Transm Infect 2001;28:633-42.

3. Mbofana FS, Brito FJ, Saifodine A, Cliff JL. Syndromic management of sexually transmitted diseases at primary care level, Mozambique. Sex Transm Infect 2002;78:E1-2.

4. Steen R, Soliman C, Mujyambwani A, et al. Notes from the field: practical issues in upgrading STD services based on experience from primary health care facilities in two Rwandan towns. Sex Transm Infect 1998;74:S159-65.

5. Harrison A, Karim SA, Floyd K, et al. Syndrome packets and health worker training improve sexually transmitted disease case management in rural South Africa: randomized controlled trial. AIDS 2000;14:2769-79.

6. Martin D, Kouman E, Masatu M, Klepp K-I. Clinicians' perspective on a training programme in syndromic management of sexually transmitted infections in Northern Tanzania. Int J STD AIDS 2005;16:697-701.

7. Hanson S, Engvall J, Sunkutu RM, et al. Case management and patient reactions: a study of STD care in a province of Zambia. Int J STD AIDS 1997;8:320-8.

8. World Health Organization. Guidelines for the management and treatment of sexually transmitted diseases, 2003. http://www.who.int/reproductive-health/ publications/mngt_stis/ (accessed 8 Jan 2008).

9. Ministry of Health of Botswana. Report on Studies on the Aetiology of Sexually Transmitted Diseases in Botswana; studies conducted by the National AIDS Control Program of the Ministry of Health of Botswana and the STD Research Unit, South African Institute for Medical Research, Johannesburg. Botswana, 1993.

10. University of Oslo. Report on the Aetiology and Prevalence of STDs and Risk Factors in Botswana; a national survey on women by STD Programme and NHL of Ministry of Health, NIR of University of Botswana and Department of Community Medicine of University of Oslo. Botswana, 1997.

11. Paz-Bailey G, Rahman M, Chen C, et al. Changes in etiology of sexually transmitted diseases in Botswana between 1993 and 2002: implications for the clinical management of genital ulcer disease. Clin Infect Dis 2005;41:1304-12.

12. Botswana National AIDS coordinating agency (NACA). Botswana 2001 HIV sero-prevalence sentinel survey amongst pregnant women and men with sexually transmitted infections; A technical report - December 2001. Gaborone: NACA, 2001. http://www.naca.gov.bw (accessed 12 0ct 2006).
13. Seipone K, Ntumy R, Smith M, et al. Introduction of routine HIV testing in prenatal care. MMWR Morb Mortal Wkly Rep 2004;53:1083-6.

14. Steen TW, Seipone K, Gomez FDLH, et al. Two and a half years of routine HIV testing in Botswana. J Acquir Immune Defic Syndr 2007;44:484-8.

15. Botswana Central Statistics Office. Population of towns, villages, and associated localities in August 2001. Gaborone: Botswana Department of Printing and Publishing Service, 2002. http://www.cso.gov.bw/html/census/tab_cens1.2.html laccessed 23 Aug 2006).

16. Botswana Ministry of Health, BOTUSA, I-TECH. Management of Sexually Transmitted Infections: STI Management Course-Facilitator's Guide. June 2005. http://www.go2itech.org/itech?page $=$ db-02-008id $=50926$ gbkto $=$ dbsearch\&query = Mnagement\%20of\%20Sexually\%20Transmitted\%20Infections\&start (accessed 8 Jan 2008).

17. Botswana Ministry of Health, BOTUSA, I-TECH. Management of Sexually Transmitted Infections: STI Management Course-Participant's Handbook. http:// www.go2itech.org/itech?page $=\mathrm{db}-02$-00\&id $=50926$-02\&type $=$ \&bkto $=$ db-0200\&bktopost $=$ \&p-name $=$ \&p-value (accessed 8 Jan 2008)

18. Botswana Ministry of Health, BOTUSA, I-TECH. Management of Sexually Transmitted Infections: Reference Manual for Health Workers. June 2005. http:// www.go2itech.org/itech?page $=$ db-02-00धid $=50926-02 \&$ type $=$ qbkto $=$ db-0200\&bktopost $=$ \&p-name $=$ \&p-value (accessed 8 Jan 2008).

19. Botswana Ministry of Health, BOTUSA, I-TECH. Management of Sexually Transmitted Infections: Clinical Photo Reference Guide. June 2005. http://www. go2itech.org/itech?page $=$ db-02-00\&id $=$ 51040\&type $=$ \&bkto $=\mathrm{db}-02$ 00\&bktopost $=\& p$-name $=\& p$-value (accessed 8 Jan 2008)

20. McNutt L-A, Wu C, Xue X, Hafner JP. Estimating the relative risk in cohort studies and clinical trials of common outcomes. Am J Epidemiol 2003;157:940-3.

21. Zou G. A modified Poisson regression approach to prospective studies with binary data. Am J Epidemiol 2004;159:702-6.

22. World Health Organization/UNAIDS. UNAIDS/WHO policy statement on HIV testing. WHO/UNAIDS, 2004. http://www.who.int/ethics/topics/en/ hivtestingpolicy_who_unaids_en_2004.pdf (accessed 8 Jan 2008).

23. Day S, Lakhank $D$, Hankins $\bar{M}$, Rodgers $C A$. Improving uptake of HIV testing in patients with confirmed STI. Int J STD AIDS 2004;15:626-8.

24. O'Hara HB, Voeten HACM, Kuperus AG, et al. Quality of health education during STD case management in Nairobi, Kenya. Int J STD AIDS 2001;12:315-23.

25. Franco LM, Daly CC, Chilongozi D, Dallabetta G. Quality of case management of sexually transmitted diseases: comparison of the methods for assessing the performance of providers. Bull WHO 2997;75:523-32.

26. Mathews C, van Rensburg A, Schierhout G, et al. An assessment of care provided by a public sector STD clinic in Cape Town. Int J STD AIDS 1998; 9:689-94.

27. Vuylsteke B, Traore M, Mah-Bi G, et al. Quality of sexually transmitted infections services for female sex workers in Abidjan, Cote d'Ivoire. Trop Med Int Health 2004; 9:638-43

28. Luck J, Peabody JW. Using standardized patients to measure physicians' practice: validation study using audio recordings. BMJ 2002;325:679-83.

29. Ware JE Jr, Hayes RD. Methods for measuring patient satisfaction with specific medical encounters. Med Care 1988;26:393-401.

30. Pascoe GC. Patient satisfaction in primary health care: a literature review and analysis. Eval Program Plann 1983;6:185-210. 